VALIDATION OF THE X-RAY FLUORESCENCE (FRX-ED) ANALYTICAL METHOD FOR THE DETERMINATION OF METALS IN SOILS AT COLQUENCHA MUNICIPALITY

\section{VALIDACIÓN DEL MÉTODO ANALÍTICO DE FLUORESCENCIA DE RAYOS X (FRX-ED) PARA LA DETERMINACIÓN DE METALES EN SUELOS DEL MUNICIPIO DE COLQUENCHA}

Full original article

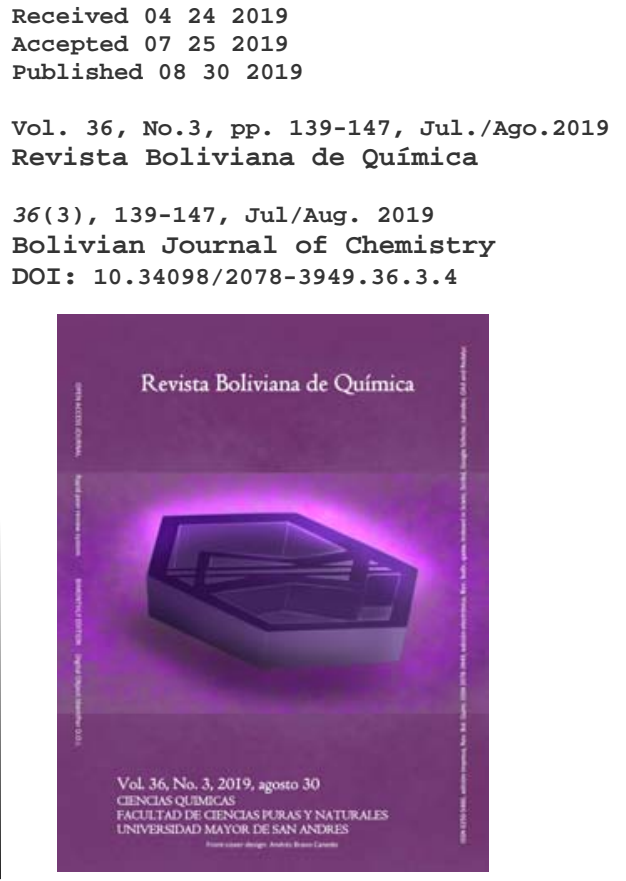

Peer-reviewed

Maria Isabel Chambi Tapia ${ }^{1, *}$, Magaly Rosio Cori Mamani ${ }^{1}$, Jorge Chungara Castro ${ }^{2}$, Samuel Fernandez Alcazar ${ }^{2}$, Oswaldo Eduardo Ramos Ramos ${ }^{1}$

${ }^{1}$ Department of Chemical Sciences, Instituto de Investigaciones Químicas IIQ, School of Pure and Natural Sciences FCPN, Universidad Mayor de San Andrés UMSA, P.O. Box 303, Calle Andrés Bello s/n Edificio IIQ, Campus Cota Cota, Phone +59122795878, La Paz, Bolivia, http://cienciasquimicas.umsa.bo/

${ }^{2}$ Agencia Boliviana de Energía Nuclear ABEN, Centro de Investigación y Aplicaciones Nucleares CIAN, Calle Jaime Mendoza Nro. 987, Esq. Peñaranda, Edificio Torre Soleil (Calacoto), Phone 59122127178, fax +59122129754 Av. 6 de agosto N²905 Phone +59122433481, comunicacion@aben.gob.bo, Casilla 4821, La Paz, Bolivia, http://www.aben.gob.bo/es/contacto

Keywords: Fluorescencia RX, Metales pesados, Suelos agrícolas, ISO 17025.

Palabras clave: X-R Fluorescence, Heavy metals, Agricultural soils, ISO 17025.

\title{
ABSTRACT
}

The analytical method by the technique of X-ray Fluorescence with dispersive energy (FRX-ED) for the determination of the concentrations of $\mathrm{Cu}, \mathrm{Fe}, \mathrm{Mn}, \mathrm{Ni}$ and $\mathrm{Zn}$, has been validated with statistical tools in soil samples extracted from calicate soils of the Colquencha municipality. The FRX-ED technique does not require prior treatment in the preparation of standards, reference materials or soil samples, which are analyzed in the form of tablets. The quantification of the $\mathrm{Cu}, \mathrm{Fe}, \mathrm{Mn}, \mathrm{Ni}$ and $\mathrm{Zn}$ metals is determined through the QXAS software and contrasted with the SOIL-7 certified reference material and the MAPEP-7 intercomparison material. Validated parameters for $\mathrm{Cu}, \mathrm{Fe}, \mathrm{Mn}, \mathrm{Ni}$ and $\mathrm{Zn}$ compared to SOIL-7 and $\mathrm{Ni}$ and $\mathrm{Zn}$ compared to MAPEP-7 that include detection limit (LOD) and quantification (LOQ) are within the acceptance criteria. Reproducibility and repeatability have error values that are below $5 \%$ for $\mathrm{Cu}, \mathrm{Mn}$ and $\mathrm{Zn}$, while Fe showed values below $1 \%$. The accuracy evaluated with the student $\mathrm{t}$ (one tail) does not present a significant difference for $\mathrm{Cu}, \mathrm{Mn}$ and $\mathrm{Zn}$, excepting Fe. The results 
María Isabel Chambi Tapia et al. RBQ Vol. 36, No.3, pp. 139-147, 2019

obtained by FRX-ED showed us that the concentrations of the metals $\mathrm{Cu}, \mathrm{Fe}, \mathrm{Mn}$ and $\mathrm{Zn}$ in the soils are within the maximum permissible limits, according to the data reported in international studies, having established this, that the soils of the municipality of Colquencha are suitable for cultivation.

\section{*Corresponding author: iisatkml@gmail.com}

\section{RESUMEN}

El método analítico por la técnica de Fluorescencia de rayos X con energía dispersiva (FRX-ED) para la determinación de las concentraciones de $\mathrm{Cu}, \mathrm{Fe}, \mathrm{Mn}$, $\mathrm{Ni}$ y $\mathrm{Zn}$, ha sido validado con herramientas estadísticas en muestras de suelos extraídas de calicatas del municipio de Colquencha. La técnica FRX-ED no requiere de tratamiento previo en la preparación de los estándares, de los materiales de referencia ni de las muestras de suelos, las cuales son analizadas en forma de pastillas. La cuantificación de los metales $\mathrm{Cu}, \mathrm{Fe}, \mathrm{Mn}, \mathrm{Ni}$ y $\mathrm{Zn}$ es determinada a través del software de QXAS y contrastados con el material de referencia certificada SOIL-7 y el material de intercomparación MAPEP-7.

Los parámetros validados para $\mathrm{Cu}, \mathrm{Fe}, \mathrm{Mn}$, Ni y $\mathrm{Zn}$ comparados con SOIL-7 y Ni y $\mathrm{Zn}$ comparados con MAPEP-7 que incluyen límite de detección (LOD) y de cuantificación (LOQ) se encuentran dentro de los criterios de aceptación. La reproducibilidad y repetitividad presentan valores de error que están por debajo del $5 \%$ para $\mathrm{Cu}$, Mn y Zn, mientras que el Fe muestra valores por debajo del $1 \%$. La exactitud evaluada con la t student (una cola) no presenta diferencia significativa para $\mathrm{Cu}, \mathrm{Mn}$ y $\mathrm{Zn}$, excepto para el $\mathrm{Fe}$. Los resultados obtenidos por FRX-ED nos muestran que las concentraciones de los metales $\mathrm{Cu}, \mathrm{Fe}, \mathrm{Mn}$ y $\mathrm{Zn}$ en los suelos están dentro de los límites máximos permisibles, según los datos reportados en estudios internacionales, habiéndose establecido así, que los suelos del municipio de Colquencha son aptos para su cultivo.

\section{INTRODUCCIÓN}

Los micronutrientes $(\mathrm{Cu}, \mathrm{Fe}, \mathrm{Mn}, \mathrm{Ni}$ y $\mathrm{Zn})$ presentes en suelos en pequeñas cantidades son importantes para la producción agrícola ya que inciden en una serie de procesos relacionados al metabolismo y transferencia de energía., Estos micronutrientes en concentraciones altas pueden ser tóxicos cuando forman parte de los cultivos [2]. El presente trabajo presenta la validación de un método analítico semicuantitativo por comparación con material de referencia (MRC; SOIL-7) y material de intercomparación (Mixed Analyte Perfomance Evaluation Program, MAPEP-00-S7) de algunos micronutrientes ( $\mathrm{Cu}, \mathrm{Fe}, \mathrm{Mn}, \mathrm{Ni}$ y $\mathrm{Zn}$ ) provenientes de los diferentes horizontes de suelo (calicatas del municipio de Colquencha). Para tal efecto se ha empleado la técnica de fluorescencia de rayos $\mathrm{X}$ con energía dispersiva (FRX-ED), con la aplicación de conceptos estadísticos de la ISO/IEC 17025. La contrastación de resultados con estudios previos empleando esta técnica no fue posible debido a que este estudio es uno de los primeros que utiliza la técnica de FRX-ED y su aplicación a estudios en suelos agrícolas en Bolivia. El área de estudio fue el municipio de Colquencha (Provincia Aroma, departamento de La Paz, Bolivia) entre los $16^{\circ} 52^{\prime}$ a $17^{\circ} 7^{\prime}$ de latitud Sur y $68^{\circ} 17^{\prime}$ a $68^{\circ} 25^{\prime}$ de longitud Oeste, a una altura promedio de 3890 m s.n.m. El área de estudio cuenta con cuatro cantones: Machacamarca, Colquencha, Micaya y Marquiviri (Figura 1).

\section{EXPERIMENTAL}

La validación del método fue aplicada a ocho muestras de suelos (calicatas; ancho $1 \mathrm{~m}$, largo $1 \mathrm{~m}$ y de cada uno de los horizontes) ubicadas en las aynokas (sistema tradicional de rotación de cultivos) del municipio de Colquencha. Las muestras fueron secadas a temperatura ambiente y tamizadas con una malla de $2 \mathrm{~mm}$ y posteriormente a través de una malla $63 \mu \mathrm{m}$ para lograr homogeneidad; las muestras tamizadas usadas para la determinación de concentraciones de $\mathrm{Cu}, \mathrm{Fe}, \mathrm{Mn}$, Ni y $\mathrm{Zn}$ fueron colocadas en bolsas tipo ziploc ${ }^{\circledR}$.

\section{Instrumentos}

La técnica de fluorescencia de rayos X (FRX) es una técnica no destructiva utilizada para la evaluación cualitativa y cuantitativa de varios tipos de muestras (agua, suelos, sedimentos, plantas y otros) [6]. El instrumento FRX-ED consta de un generador de Rayos-X ITAL STRUCTURES modelo COMPACT 3k5, con rangos operativos de 
ISSN 2078-3949 Rev, boliv. quim. Electronic edition

Published 0830 2019; DOI:10.34098/2078-3949.36.3.4 María Isabel Chambi Tapia et al. RBQ Vol. 36, No.3, pp. 139-147, 2019

voltaje y amperaje de $40 \mathrm{kV}$ y $20 \mathrm{~mA}$ respectivamente, aplicando tiempos de irradiación de 200 segundos. La cuantificación de las concentraciones de los metales $\mathrm{Cu}, \mathrm{Fe}, \mathrm{Mn}$, Ni y $\mathrm{Zn}$ fue determinada por el software Quantitative X Ray Analysis System (QXAS 1994, versión 3.6). El instrumento se encuentra en las instalaciones de Centro de Investigación de Aplicación Nuclear CIAN de la Agencia Boliviana de Energía Nuclear ABEN.

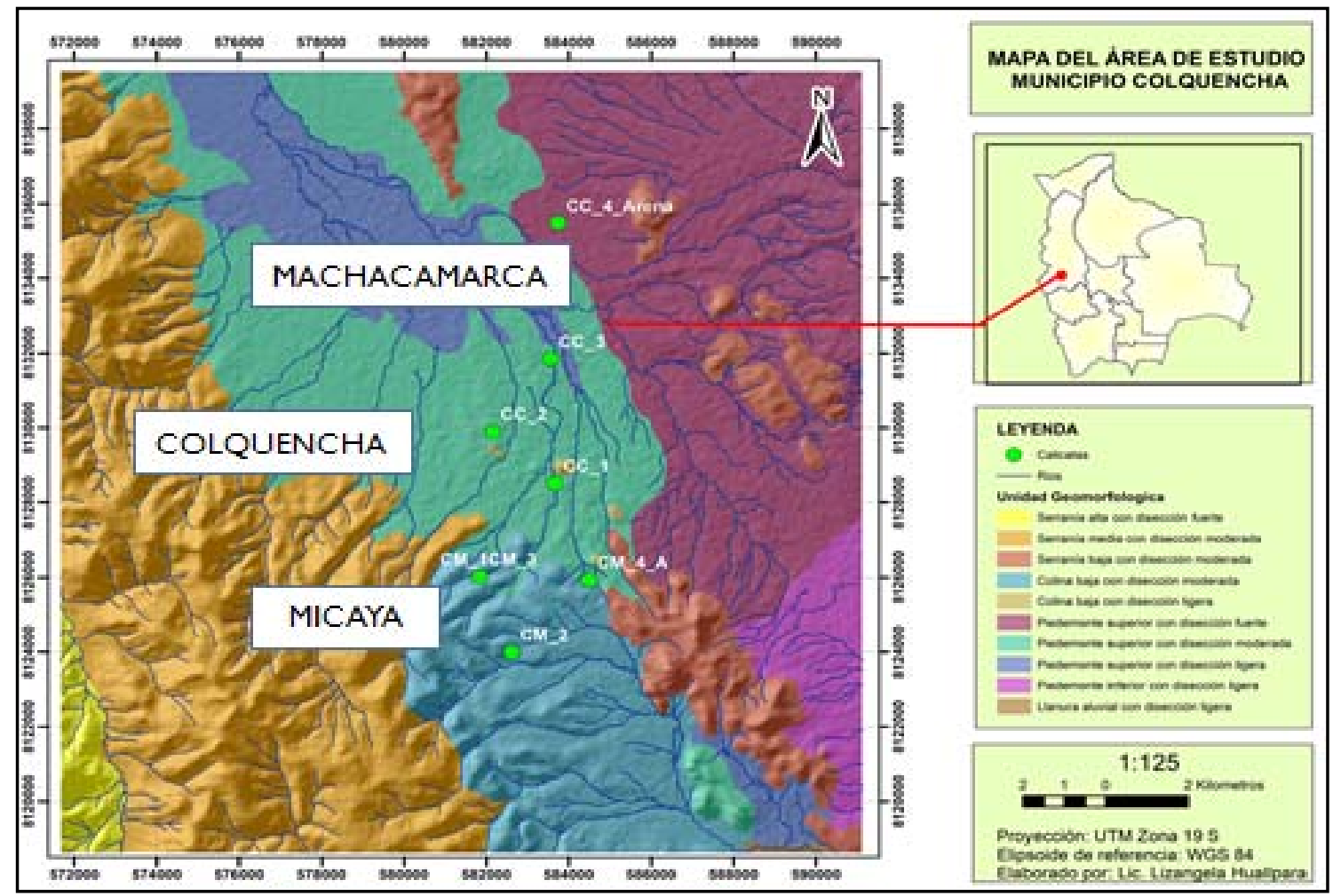

Figura 1: Ubicación del Municipio de Colquencha y los puntos de muestreo de las calicatas de suelos

\section{Materiales y métodos}

La preparación de pastillas de muestras, blancos y estándares fue realizada por pesada del material tamizado en una balanza analítica (Sartorius $\pm 0,0005 \mathrm{~g}$ ). La preparación de la pastilla del material de referencia certificado (MRC; SOIL-7) y la del material de intercomparación (MDI, MAPEP-S7) fue realizada pesando $1,5 \mathrm{~g}$ de cada MRC o muestra de suelo (calicatas) con $0,5 \mathrm{~g}$ de un componente inerte almidón (p.a.) a temperatura ambiente, homogenizando en un mortero y prensado a 10 toneladas de presión por 30 segundos; la preparación de pastillas de los blancos; almidón comercial (maicena comercial Kris ${ }^{\circledR}$ ) y almidón (p.a.) fue realizada pesando 2,0 $\mathrm{g}$ de cada blanco en las mismas condiciones.

La limpieza del mortero es realizada moliendo vidrio hasta obtener un polvo fino para eliminar los restos de partículas de material (suelo, MDI y/o MRC) que queda en el mortero para luego ser desechado. Se lavó luego el mortero con agua destilada y desionizada y con alcohol etílico (96\%), mientras que la prensa se lavó con alcohol etílico (96\%) que es un procedimiento interno del CIAN-ABEN.

Los parámetros de validación fueron: limite detección (LOD) y cuantificación (LOQ), precisión, exactitud y robustez que fueron determinados según el procedimiento ISO 17025 y el Eurochem (2005). El LOD y LOQ se determinó a partir de un material inerte en este caso almidón (p.a.) pesando 2,0 $\mathrm{g}$ de masa total y realizando seis lecturas de cada blanco y aplicando la ecuación de LOD según la ecuación EC-1 (tabla A1, anexo) y LOQ según la ecuación EC-2 (tabla A1).

La precisión fue establecida en términos de la repetitividad y reproducibilidad aplicando las ecuaciones EC-3 y EC-4 (tabla A1) respectivamente, empleando el MRC (SOIL-7) y el MDI (MAPEP-S7) y una muestra de suelo (CM-4, aynoka de Micaya), preparando seis pastillas de MRC, MDI y blancos respectivos. La repetitividad se 
María Isabel Chambi Tapia et al. RBQ Vol. 36, No.3, pp. 139-147, 2019

obtuvo preparando e irradiando las pastillas por un mismo analista, el mismo día y utilizando el mismo instrumento. La reproducibilidad fue determinada por diferentes analistas tanto en el preparado como en la irradición de las pastillas en distintas fechas, utilizando el mismo instrumento pero a diferentes temperaturas ambientales (cumpliendo los lineamientos de ISO 17025).

La exactitud fue determinada en términos del sesgo, empleando el MRC y MDI y aplicando la ecuación EC-5 (tabla A1). El grado de concordancia entre el resultado obtenido experimentalmente y el valor del MRC y MDI determinado aplicando la t-student (con un nivel de confianza del 95\% evaluando para ello el t-critico o t-tabulado ( $t_{\text {tab }}$ para un grado de libertad de $\left.v=n-1\right)$, dio una cola y un valor de $\alpha=0,05$.

El porcentaje de recuperación $(\% \mathrm{R})$ fue determinado para el analito $\mathrm{Cu}$, pesando $1,498 \mathrm{~g}$ de muestra de suelo CM-4 con 0,002 g de $\mathrm{CuO}$ al $99 \%$ de pureza y 0,5 de almidón (p.a). Se prepararon seis pastillas, se irradiaron muestras adicionadas con el analito $\mathrm{Cu}$ y sin adición de ningún analito. El \% R fue determinado aplicando la ecuación EC-7 (tabla A1), la magnitud de la diferencia entre el \%R obtenido y el $10 \%$ de recuperación fue determinado aplicando la $t$-student con un nivel de confianza del $95 \%$ (calculada la $t_{\text {cal }}$ con grados de libertad de $n-1$ $\left.t_{\text {tab }}=2,015\right)$. El estudio de robustez implicó realizar variaciones respecto al método establecido, como ser tiempo de irradiación: 200 s y 500 s, variación de la corriente: 5, 10, 15 y $20 \mathrm{~mA}$, as \{i como de cambio de analista; material inerte: almidón (p.a.) y almidón comercial y temperatura ambiente. Para la determinación de los parámetros de validación se realizaron seis lecturas del material de referencia certificado (SOIL-7), material de intercomparación (MAPEP-S7), muestra de suelo CM-4 y un blanco de matriz (almidón).

\section{RESULTADOS Y DISCUSIÓN}

La validación del método analítico comprende la realización de varias pruebas con el material de referencia certificada (MRC) y con el material de intercomparación (MDI). Para demostrar los alcances y limitaciones que tiene el método analítico, éste ha sido evaluado por medio de un análisis estadístico para establecer sus límites de aplicación.

El LOD y LOQ determinado por la metodología para el caso del $\mathrm{Cu}$, Ni y $\mathrm{Zn}$, proporcionan valores menores en relación al Fe y Mn; por lo tanto, se indica que el equipo de FRX-ED tiene menor sensibilidad analítica para la determinación de estos dos analitos (Tabla 1).

Tabla 1. Valores de los límites de detección (LOD) y límites de cuantificación (LOQ)

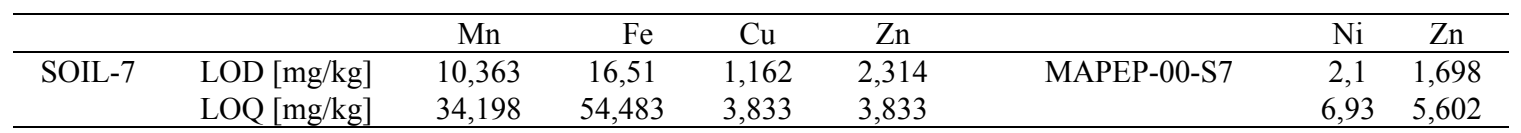

\section{Precisión (repetitividad y reproducibilidad)}

Los resultados obtenidos muestran que la repetitividad $\left(\% \mathrm{CV}_{\mathrm{r}}\right)$ para los metales de $\mathrm{Cu}, \mathrm{Mn}, \mathrm{Zn}$ (SOIL-7) y Ni y $\mathrm{Zn}$ (MAPEP-S7) son $>$ al 1\%, excepto para el Fe (SOIL-7) que es < al 1\% (Tabla 2); dado que estos dos materiales fueron evaluados en términos de homogeneidad (i.e. Reference sheet MRC, IAEA-SOIL-7) podemos atribuir las diferencias a errores aleatorios. La determinación de Fe presenta un pico intenso (elevada concentración) donde la cuantificación de su concentración es poco influenciada por las pequeñas variaciones de concentración, en comparación con los otros analitos $(\mathrm{Cu}, \mathrm{Mn}, \mathrm{Ni}$ y $\mathrm{Zn}$ ) cuyas concentraciones presentan pequeños picos donde una pequeña variación influye en el resultado final. Los resultados de reproducibilidad muestran que ${ }_{0} \mathrm{CV}_{\mathrm{R}}$ para $\mathrm{Cu}, \mathrm{Fe}$, $\mathrm{Mn}$ y $\mathrm{Zn}$ determinados por comparación con SOIL-7 y $\% \mathrm{CV}_{\mathrm{R}}$ para $\mathrm{Ni}$, Zn determinados por comparación con MAPEP-S7 son > al 1\% (rango 2,50\%-7,98\%), indicando así que existe mayor error producido en la preparación del material de referencia y en el material de intercomparación por diferentes analistas, diferentes días y temperatura (Tabla 2); los resultados también están influenciados por el tamaño de pico de los dos materiales. Los resultados obtenidos muestran que los valores de \%CVr \& \%CVR son $<10 \%$ demostrando el cumplimiento de los criterios de aceptación.

Exactitud/sesgo y \% de recuperación 
María Isabel Chambi Tapia et al. RBQ Vol. 36, No.3, pp. 139-147, 2019

Los resultados de $t_{\text {cal }}$ obtenidos para los analitos $\mathrm{Cu}$, Mn y $\mathrm{Zn}$ (SOIL-7) y Ni, Zn (MAPEP-S7) son $<t_{\text {tab }}$ indicando que existe una diferencia mínima entre el valor experimental y el valor del MRC; por lo tanto, la veracidad es aceptable [7]. En cambio, para Fe (SOIL-7) el $t_{\text {cal }}>t_{\text {tab }}$ indicando que existe una diferencia significativa entre el valor experimental y el valor de MRC; por lo tanto, la veracidad no es aceptable [7] (Tabla 2).

El resultado del \% de recuperación de una muestra adicionada con un estándar de $\mathrm{Cu}$ de $791 \mathrm{mg} / \mathrm{kg}$, muestra que la concentración es expresada en porcentaje en masa $0,0791 \%$ de $\mathrm{Cu}$ lo cual es aceptable para el nivel medido en la matriz analizada según los criterios de la AOAC (Association of Official Analytical Chemists) con un valor de 95 a $100 \%$ de recuperación y con un error de $5 \%$ (Tabla 2). El resultado de $t_{\text {cal }}$ obtenido para el \% de recuperación del $\mathrm{Cu}$ es menor al $\mathrm{t}_{\text {tab }}$ la cual indica que no existe diferencia significativa.

Tabla 2. Parámetros de validación del MRC (SOIL-7) certificado por la IAEA y del MDI (MAPEP-00-S7)

\begin{tabular}{|c|c|c|c|}
\hline \multicolumn{4}{|c|}{$\begin{array}{c}\text { Precisión y Exactitud } \\
\text { SOIL-7 }\end{array}$} \\
\hline Elementos & $\begin{array}{c}\text { Repetibilidad } \\
\text { \% CVr }\end{array}$ & $\begin{array}{c}\text { Reproducibilidad } \\
\% \text { CVR }\end{array}$ & $\begin{array}{c}\text { Exactitud } \\
\text { Sesgo }\end{array}$ \\
\hline $\mathrm{Mn}$ & 4,513 & 7,120 & 61,580 \\
\hline $\mathrm{Fe}$ & 0,276 & 7,980 & 1141,667 \\
\hline $\mathrm{Cu}$ & 5,004 & 3,590 & 0,048 \\
\hline $\mathrm{Zn}$ & 2,343 & 4,286 & $-4,631$ \\
\hline \multicolumn{4}{|c|}{ MAPEP-00-S7 } \\
\hline $\mathrm{Ni}$ & 8,188 & 4,620 & 5,916 \\
\hline $\mathrm{Zn}$ & 3,300 & 2,506 & 1,641 \\
\hline \multicolumn{4}{|c|}{ Criterios de aceptación } \\
\hline \multicolumn{2}{|c|}{$\% \mathrm{r} \& \mathrm{R}$} & $\% \mathrm{r} \& \mathrm{R}<10 \%$ & Cumple \\
\hline \multicolumn{2}{|c|}{ Exactitud: $\mathrm{Mn}, \mathrm{Ni}, \mathrm{Cu}$ y $\mathrm{Zn}$} & $t_{\exp }<\mathrm{t}_{\mathrm{tab}}$ & Cumple \\
\hline \multicolumn{2}{|c|}{ Exactitud: Fe } & $t_{\exp }>t_{t a b}$ & no cumple \\
\hline
\end{tabular}

\section{Robustez}

La robustez es determinada utilizando una muestra de suelo en la calicata CM-4, en donde se ha variado la corriente y el tiempo de irradiación. La respuesta de la variación de la corriente y el tiempo de irradiación (Figura 2) se evidencia con el incremento el área de cada pico de los analitos presentes en la muestra (CM-4), indicando que existe una relación directa entre la corriente y el tiempo de irradiación con la concentración.

La variación de los blancos utilizando como aglomerante almidón soluble p.a. y almidón comercial fue determinada irradiando y observando la intensidad de los picos de $\mathrm{Cu}$, estos son superiores al pico de la muestra, esto puede ser debido a que se ha irradiado mayor cantidad de masa $(2,0 \mathrm{~g}$ del blanco y no $0,5 \mathrm{~g})$ como es habitual, indicando que es importante restar la concentración del blanco con la muestra sobre todo para el $\mathrm{Cu}$ ya que puede interferir durante la determinación (Figura 3).

\section{Concentración de analitos en las calicatas}

La concentracion de Mn en los suelos de las calicatas de Colquencha, Machacamarca, Marquiviri y Micaya es mayor en la parte superficial que a mayor profundidad, esto debido al $\mathrm{pH}$ bajo de la superficie. La concentración de Fe (expresada en \% en masa) es alta, principalmente debido a su abundancia en la corteza terrestre y es absorbida en pequeñas cantidades por los cultivos por la muy baja solubilidad de la mayoría de sus compuestos [1]. La acumulación de $\mathrm{Fe}$ es irregular en diferentes capas debido a que el exceso de $\mathrm{Mn}$ en el suelo contribuye a la deficiencia de $\mathrm{Fe}$ (Figura 4).

$\mathrm{La}$ acumulación de $\mathrm{Cu}$ y $\mathrm{Zn}$ en los suelos de las calicatas es irregular debido al tipo de cultivo y sistema de rotación de cultivos que existe en la zona de estudio. Por otra parte, la fertilización intensiva con $\mathrm{N}$ y $\mathrm{P}$ puede agravar la falta de $\mathrm{Cu}$. La baja movilidad del $\mathrm{Zn}$ en los suelos depende del medio y de la forma en la que esté presente este micronutriente (Figura 5).

Los rangos de concentración de los analitos son: $\mathrm{Cu} 3,481 \pm 1,173 \mathrm{mg} / \mathrm{kg}$ a $8,579 \pm 1,373 \mathrm{mg} / \mathrm{kg}$ y Zn 48,646 \pm $3,062 \mathrm{mg} / \mathrm{kg}$ a $129,932 \pm 7,856 \mathrm{mg} / \mathrm{kg}$ en los suelos de las calicatas, estos valores se encuentran dentro de los límites

$$
\begin{array}{ccc}
\text { Downloadable from: Revista Boliviana } 143 \text { de Química. Volumen } 36 \mathrm{~N}^{\circ} 3 \text {. Año } 2019 \\
\text { http://www.bolivianchemistryjournal.org, http://www.scribd.com/bolivianjournalofchemistry }
\end{array}
$$


María Isabel Chambi Tapia et al. RBQ Vol. 36, No.3, pp. 139-147, 2019

máximos permisibles según los datos reportados (Tabla A2) por Bowie y Thornton (1985) y la ley de gestión ambiental del Ecuador (2005) siendo suelos aptos para el cultivo.

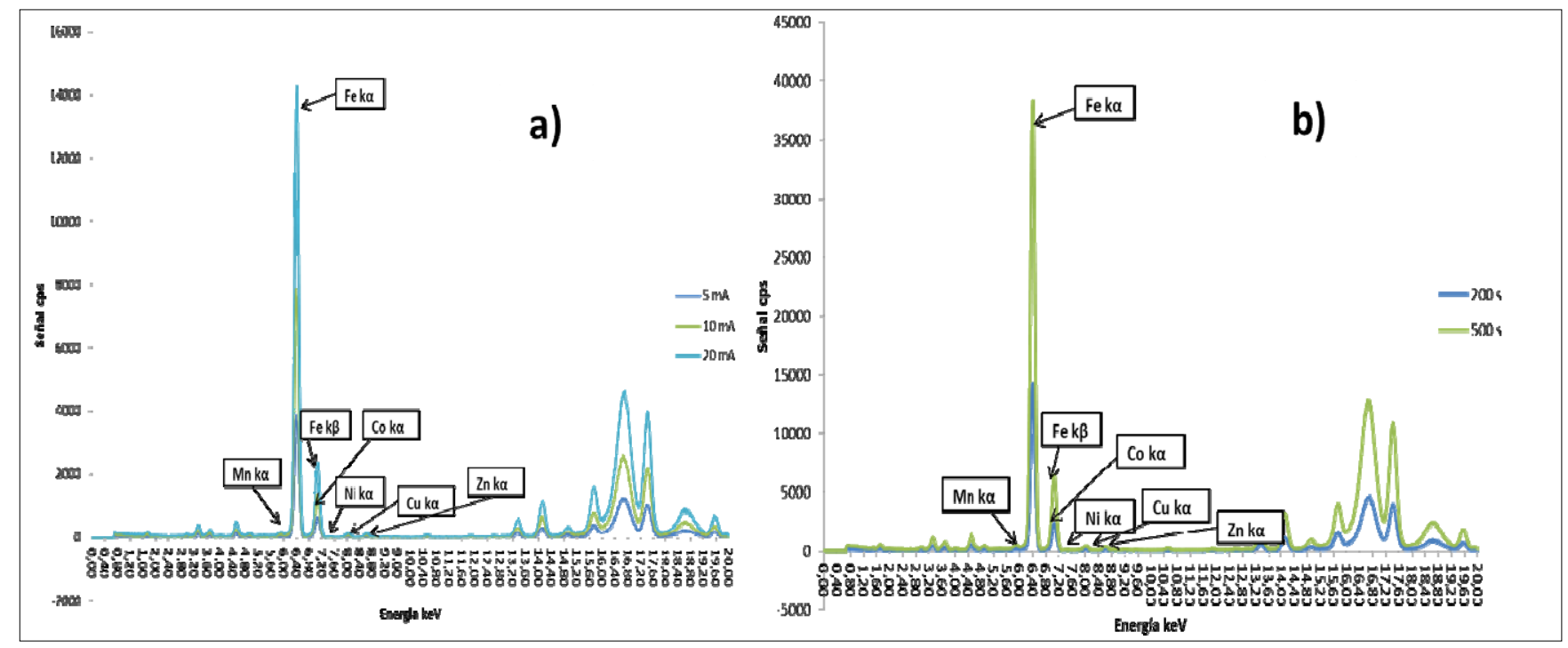

Figura 2. a) Efecto de la variación de la corriente en $m V$ en la muestra (CM-4) y b) Efecto de la variación del tiempo de irradiación en la muestra (CM-4)

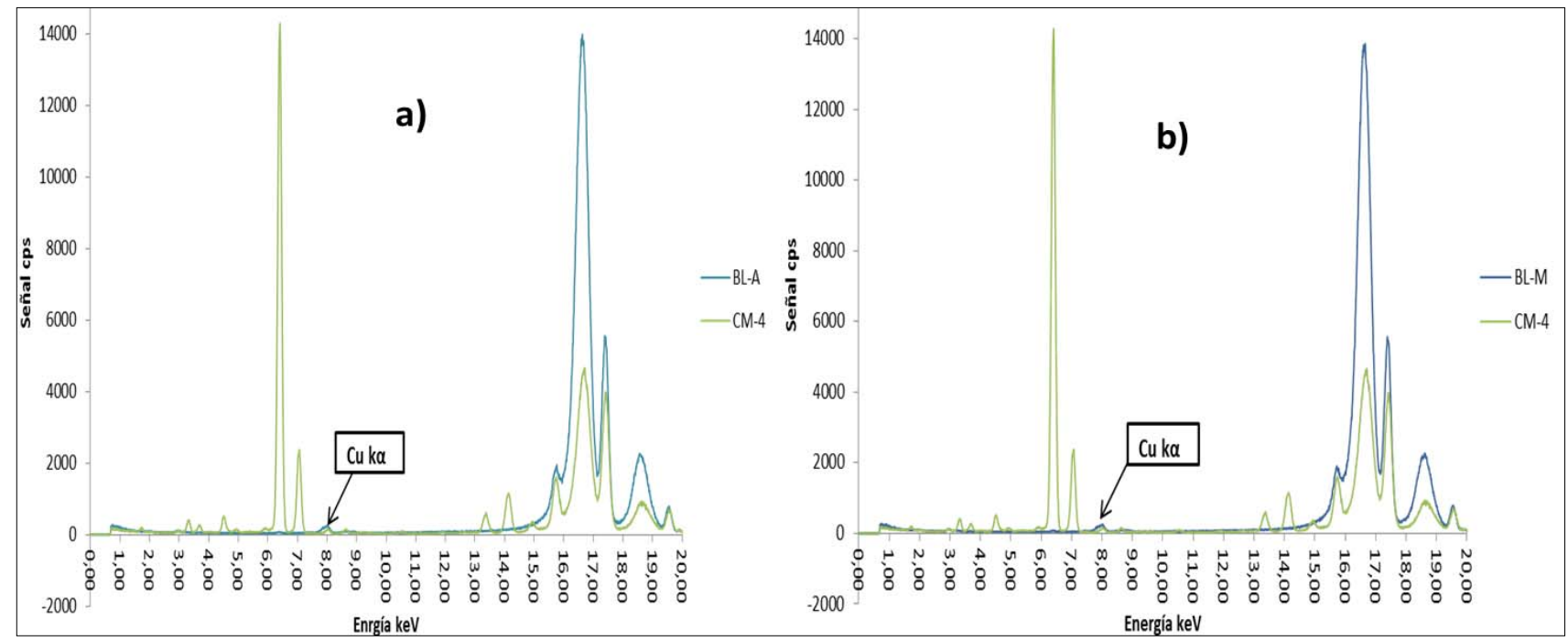

Figura 3. a) Espectro de una muestra (CM-4) y blanco (Almodón soluble) y b) Espectro de una muestra (CM-4) y blanco (Maicena Kris)

\section{CONCLUSIONES}

El método validado fue aplicado a ocho muestras de calicatas localizadas en las aynokas del municipio de Colquencha para su posterior determinación de las concentraciones de $\mathrm{Cu}, \mathrm{Fe}, \mathrm{Mn}$, Ni y Zn.

Los parámetros de validación de $\mathrm{Cu}, \mathrm{Fe}, \mathrm{Mn}, \mathrm{Ni}$ y $\mathrm{Zn}$ por comparación directa con un material de referencia certificada SOIL-7 presentan límites de detección (LOD) y cuantificación (LOQ) dentro de los criterios de aceptación, con porcentajes de reproducibilidad y repetitividad por debajo del $5 \%$ excepto para el $\mathrm{Fe}$ que se encuentra por debajo del 1\%. El sesgo evaluado con la t-student (una cola) no presenta diferencia significativa excepto para el $\mathrm{Fe}$. Los parámetros de validación de $\mathrm{Ni}$ y $\mathrm{Zn}$ por comparación directa con un material de Downloadable from: Revista Boliviana 144 de Química. Volumen 36 Nº3. Año 2019 
María Isabel Chambi Tapia et al. RBQ Vol. 36, No.3, pp. 139-147, 2019

intercomparación MAPEP-7 presentan límites de detección y cuantificación dentro de los criterios de aceptación, el porcentaje de variación para la repetitividad y la reproducibilidad se encuentran por encima del $1 \%$. El sesgo evaluado con la t-student (una cola) no presenta una diferencia significativa.

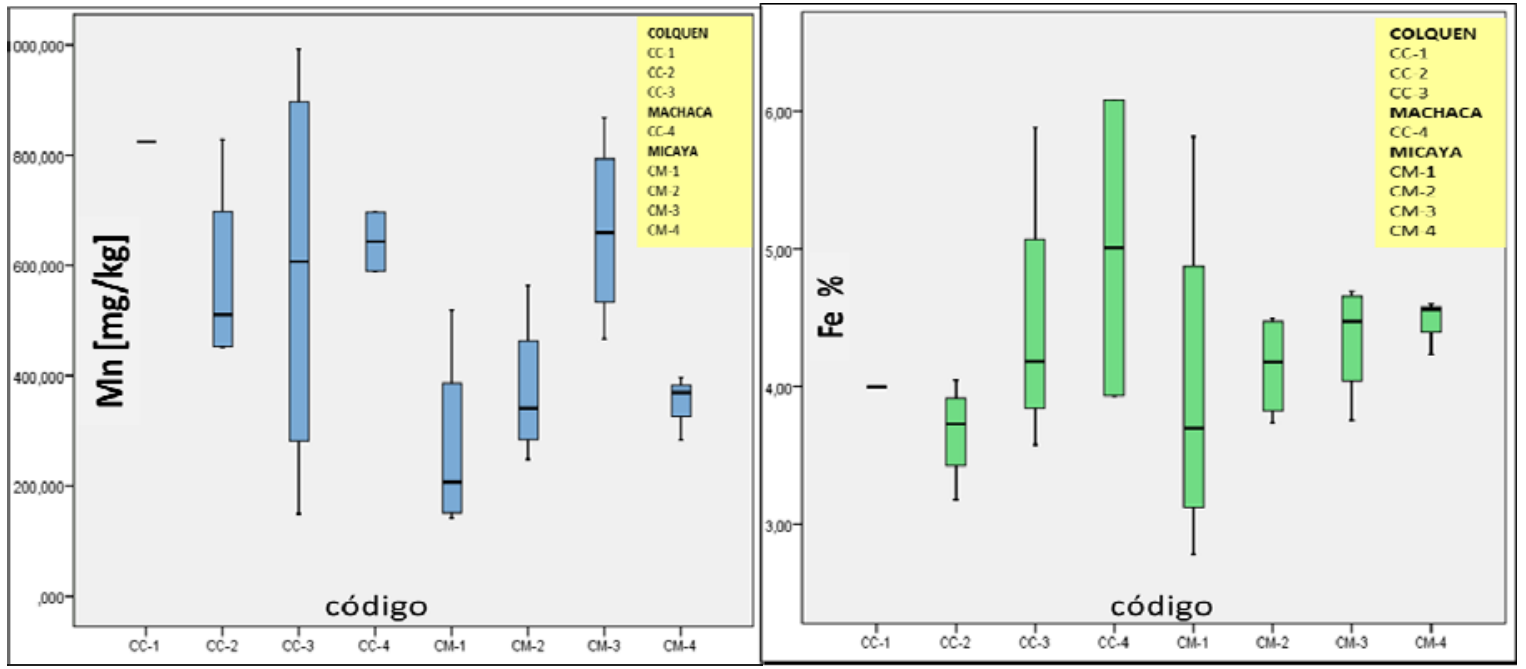

Figura 4. Concentración de Mn y Fe en suelos de calicatas de los cantones de Colquencha, Micaya y Machacamarca
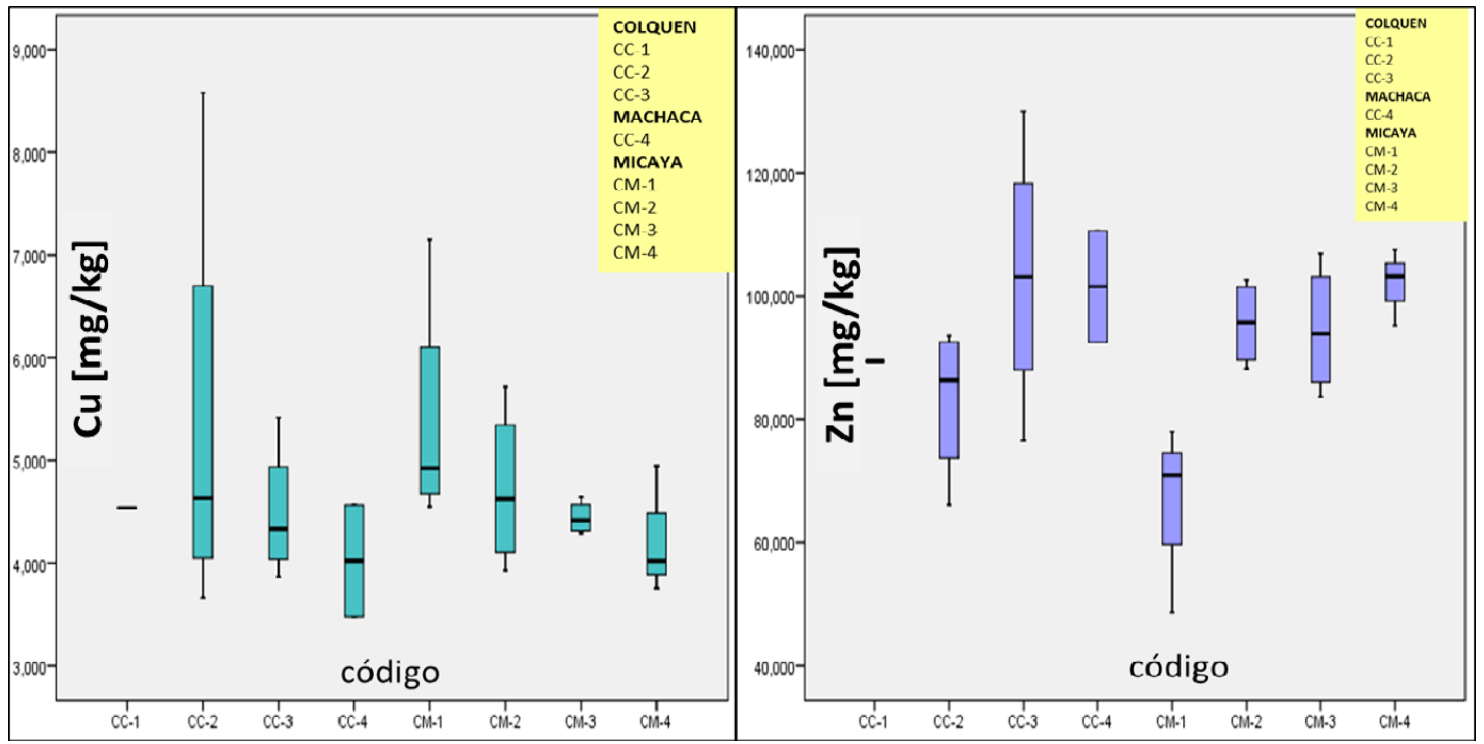

Figura 5. Concentración de Cu y Zn en suelos de calicatas de los cantones de Colquencha, Micaya y Machacamarca

Empleando la técnica de FRX-ED ha sido validado el método para la determinación de $\mathrm{Cu}, \mathrm{Fe}, \mathrm{Mn}$ y $\mathrm{Zn}$ en suelos agrícolas utilizando un material de referencia certificada (SOIL-7), también fueron validados los métodos de determinación de Ni y Zn utilizando un material de intercomparación (MAPEP-7); mientras que para Co y Ni las concentraciones se encuentran por debajo del límite de detección y no fueron considerados. Las concentraciones de los metales $(\mathrm{Cu}, \mathrm{Fe}, \mathrm{Mn}$ y $\mathrm{Zn})$ determinados por FRX - ED en suelos de calicatas del municipio de Colquencha comparando con los límites máximos permisible, presentan valores que están por debajo de los valores bibliográficos, siendo estos suelos aptos para el cultivo.

\section{RECONOCIMIENTOS}

Downloadable from: Revista Boliviana 
Al Ing. Rubén Callisaya (CIAN, ABEN) por la colaboración en el análisis de parámetros físicos del suelo; al Lic. Rolando Chuquimia Ninahuanca (Carrera de Ciencias Químicas, UMSA) por la colaboración en los análisis de muestras de suelos en el equipo de FRX-ED; a la Lic. Heidi Ruth Mamani Tola (CIAN, ABEN) por la colaboración en el tema de validación; a la Lic. Lizangela Huallpara (Carrera de Ciencias Químicas, UMSA) por la colaboración en la elaboración del mapa del área de estudio y Delia Cori en la colaboración en los análisis de las muestras de suelos. Al Dr. Leonardo Guzmán Alegría (Carrera de Ciencias Químicas, UMSA) por la colaboración en la interpretación de la parte estadística.

\section{MATERIAL SUPLEMENTARIO}

Tabla A1. Ecuaciones para la determinación de los parámetros de validación

\begin{tabular}{|c|c|c|}
\hline Nombre & Formula & Nro. Ecuación \\
\hline Límite de detección & & EC-1 \\
\hline $\begin{array}{c}\text { Límite de } \\
\text { cuantificación }\end{array}$ & 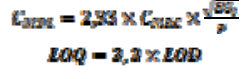 & EC-2 \\
\hline $\begin{array}{c}\text { Precisión } \\
\text { Repetibilidad }\end{array}$ & $\begin{array}{c}s_{r}=\sqrt{\frac{1}{n-1} \sum_{k=1}^{n}\left(x_{k}-\bar{x}_{i}\right)^{2}} \\
g_{r} C V_{r}=\frac{s_{r}}{\pi} \times 100\end{array}$ & EC-3 \\
\hline $\begin{array}{l}\text { Precisión } \\
\text { reproducibilidad }\end{array}$ & 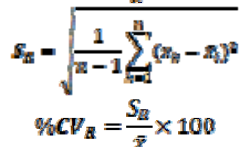 & EC-4 \\
\hline $\begin{array}{l}\text { Exactitud } \\
\text { Sesgo }\end{array}$ & $\begin{aligned} s & =\bar{x}-x_{a} \\
t_{c a l t} & =\frac{\left[x_{a z}-\bar{x}\right]}{s \times \sqrt{n}}\end{aligned}$ & EC-5 \\
\hline $\begin{array}{l}\text { Porcentaje de } \\
\text { recuperación }\end{array}$ & 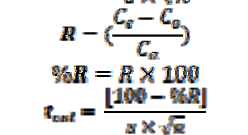 & EC-6 \\
\hline
\end{tabular}

Tabla A3. Resumen de parámetros estadísticos evaluados por SPSS

\begin{tabular}{|c|c|c|c|c|c|c|c|c|}
\hline \multicolumn{2}{|r|}{ METALES MRC-SOIL $7 \mathrm{mg} / \mathrm{kg}$} & \multirow{2}{*}{$\begin{array}{r}\text { Estadístico } \\
694,247 \\
\end{array}$} & \multirow{2}{*}{$\begin{array}{r}\text { Error estándar } \\
12,79211\end{array}$} & \multicolumn{3}{|c|}{ METALES MRC-SOIL $7 \mathrm{mg} / \mathrm{kg}$} & Estadístico & Error estándar \\
\hline \multirow{12}{*}{ Mn } & Media & & & \multicolumn{3}{|c|}{ Media } & 11,0478 & 0,22568 \\
\hline & $\begin{array}{ll}95 \% \text { de } & \text { Límite inferior } \\
\text { intervalo de } & \\
\text { confianza } & \text { Límite superior } \\
\text { para la media } & \\
\end{array}$ & $\begin{array}{l}661,3638 \\
727,1302\end{array}$ & & \multirow{11}{*}{$\mathrm{Cu}$} & $\begin{array}{l}95 \% \text { de } \\
\text { intervalo de } \\
\text { confianza } \\
\text { para la media } \\
\end{array}$ & $\begin{array}{l}\text { Límite inferior } \\
\text { Límite } \\
\text { superior }\end{array}$ & $\begin{array}{r}10,4677 \\
11,628 \\
\end{array}$ & \\
\hline & Media recortada al 5\% & 695,1564 & & & Media recortad & da al $5 \%$ & 11,0421 & \\
\hline & Mediana & 702,2165 & & & Mediana & & 10,945 & \\
\hline & Varianza & 981,828 & & & Varianza & & 0,306 & \\
\hline & Desviación estándar & 31,33414 & & & Desviación est & tándar & 0,55279 & \\
\hline & Mínimo & 643,58 & & & Mínimo & & 10,35 & \\
\hline & Máximo & 728,55 & & & Máximo & & 11,85 & \\
\hline & Rango & 84,97 & & & Rango & & 1,49 & \\
\hline & Rango intercuartil & 51,05 & & & Rango intercua & & 1,02 & \\
\hline & Asimetría & $-0,802$ & 0,845 & & Asimetría & & 0,398 & 0,845 \\
\hline & Curtosis & $-0,135$ & 1,741 & & Curtosis & & $-0,896$ & 1,741 \\
\hline \multirow{12}{*}{$\mathrm{Fe}$} & Media & 26841,6667 & 30,26733 & \multirow{12}{*}{$\mathrm{Zn}$} & Media & & 99,387 & 0,95075 \\
\hline & $\begin{array}{ll}95 \% \text { de } & \text { Límite inferior } \\
\text { intervalo de } & \\
\text { confianza } & \text { Límite superior } \\
\text { para la media } & \\
\end{array}$ & $\begin{array}{r}26763,862 \\
26919,4713\end{array}$ & & & $\begin{array}{l}95 \% \text { de } \\
\text { intervalo de } \\
\text { confianza } \\
\text { para la media } \\
\end{array}$ & $\begin{array}{l}\text { Límite inferior } \\
\text { Límite } \\
\text { superior }\end{array}$ & $\begin{array}{r}96,943 \\
101,831\end{array}$ & \\
\hline & Media recortada al 5\% & 26839,0741 & & & Media recortad & da al $5 \%$ & 99,3834 & \\
\hline & Mediana & 26840 & & & Mediana & & 99,443 & \\
\hline & $\begin{array}{l}\text { Varianza } \\
\end{array}$ & 5496,667 & & & $\begin{array}{l}\text { Varianza } \\
\end{array}$ & & 5,424 & \\
\hline & Desviación estándar & 74,13951 & & & Desviación est & tándar & 2,32886 & \\
\hline & Mínimo & 26760 & & & Mínimo & & 96,8 & \\
\hline & Máximo & 26970 & & & Máximo & & 102,04 & \\
\hline & Rango & 210 & & & Rango & & 5,24 & \\
\hline & Rango intercuartil & 112,5 & & & Rango intercua & & 4,91 & \\
\hline & Asimetría & 0,986 & 0,845 & & Asimetría & & $-0,034$ & 0,845 \\
\hline & Curtosis & 1,403 & 1,741 & & Curtosis & & $-2,374$ & 1,741 \\
\hline
\end{tabular}


Tabla A2. Límites máximos permisibles de metales de Cu y Zn

\begin{tabular}{c|cc}
\hline LIMITES MÁXIMOS PERMISIBLES & $\mathrm{Cu}[\mathrm{mg} / \mathrm{kg}]$ & $\mathrm{Zn}[\mathrm{mg} / \mathrm{kg}]$ \\
\hline LMP (Ley de Gestión Ambiental del Ecuador del 2005) & 63 & 200 \\
LMP Bowie y Thornton (1985) & $2-60$ & $25-200$ \\
\hline
\end{tabular}

Tabla A4. Valores del material de referencia certificada (MRC) y del material de intercomparación (MDI)

\begin{tabular}{l|ll}
\hline METAL & SOIL-7 & 95\% Intervalo confianza \\
\hline & $\mathrm{mg} / \mathrm{kg}$ & $\mathrm{mg} / \mathrm{kg}$ \\
$\mathrm{Mn}$ & Valores recomendados \\
$\mathrm{Fe}$ & 631 & $604-650$ \\
$\mathrm{Cu}$ & 25700 & $25200-26300$ \\
$\mathrm{Zn}$ & 11 & $9-13$ \\
& 104 & $101-113$ \\
& MAPEP-00- S7 mg/kg & \\
$\mathrm{Ni}$ & Valores de referencia \\
$\mathrm{Zn}$ & 79,7 & \\
\hline
\end{tabular}

\section{REFERENCIAS}

1. Fassbender, H. W. 1986, Química de Suelos con énfasis en suelos de América Latina Instituto Interamericano de Cooperación para la Agricultura, México, 398.

2. Lugo, G., Chavez, M., Evangelista, B., Vaca, R., del Aguila, P., Hernandez, D. 2000. Metales pesados totales y disponibles en suelos adicionados con lodos residuales después de 12 meses de aplicación, México, 2-6.

3. Llamosa, L.E., Meza, L.G., Botero, M. 2007, Estudio de repetibilidad y reproducibilidad utilizando el método de promedios y rangos para el aseguramiento de la calidad de los resultados de calibración de acuerdo con la norma técnica ISO/IEC 17025.

4. Norma Boliviana NB/ISO/IEC 17025, 2005, Requisitos generales para la competencia de los laboratorios de ensayo y calibración.

5. Eurachem, 2005. Métodos analíticos adecuados a su propósito, Guía de Laboratorio para la Validación de Métodos y temas Relacionados, México.

6. Nascimento, V.F. 1999, Técnicas Analíticas de Fluorescencia de Rayos X por Dispersión de energía (ED-XRF) y Reflexión Total (TXRF). Depto. De Ciencias Exactas/ESALQ/USP. Lab. de Instrumentación Nuclear/ CENA/USP, Sao Pablo-Brasil, 3-16.

7. Duffau B., Rojas F., Guerrero, I., Rodríguez, L., Soto, M., Aguilera, M., Sandoval, S. 2010, Validación de métodos y determinación de la incertidumbre de la medición, "Aspectos generales sobre la validación de métodos.”, Chile, 28-48. 